\title{
Prosthetic Management of Post-surgical defect in the Mandible following Marsupialization: A Case Report
}

Gurung $\mathrm{A}^{1}$, Mathema $\mathrm{SRB}^{2}$, Maskey B ${ }^{3}$, Shrestha $\mathrm{K}^{4}$, Pandey B ${ }^{5}$, Adhikari A ${ }^{6}$, Dhyako N7 , Subedi $\mathrm{S}^{8}$

1,5,6,7,8PG Resident, Department of Prosthodontics and Maxillofacial Prosthetics, People's Dental College and Hospital, Kathmandu, Nepal

${ }^{2}$ Professor, HOD, Department of Prosthodontics and Maxillofacial Prosthetics, People's Dental College and Hospital, Kathmandu, Nepal

${ }^{3}$ Assistant Professor, Department of Prosthodontics and Maxillofacial Prosthetics, People's Dental College and Hospital, Kathmandu, Nepal

${ }^{4}$ Associate Professor, Department of Prosthodontics and Maxillofacial Prosthetics, People's Dental College and Hospital, Kathmandu, Nepal

\begin{abstract}
Marsupialization is a conservative surgical approach for the surgical management of large odontogenic lesions. Such procedure often results in a post-surgical defect in the form of an intra oral opening. The patency of such defect, if not maintained can result in food accumulation which may lead to infection and thus delayed healing of the defect. Obturator is one of the modalities to facilitate closure of the resultant defect. This report presents a case where an acrylic plug obturator retained with clasp, was provided to the patient for the interim period following marsupialization on the mandibular left posterior region.
\end{abstract}

Key words: Clasp retained acrylic plug; Intraoral opening; Marsupialization; Obturator

\section{Introduction}

$\mathrm{O}$ dontogenic cysts and tumors represent a diverse group of lesions of the jaws and overlying soft tissues. The Partsch I (decompression and marsupialization) and Partsch II (enucleation and primary closure) procedures are conventional treatment modalities for benign tumors and cysts of the jaw. In cases of large lesions, marsupialization or Partsch $\mathrm{I}$ is the preferred procedure due

\section{*Corresponding Author}

Dr. Anita Gurung, PG Resident

Department of Prosthodontics and Maxillofacial

Prosthetics, People's Dental College and Hospital, Kathmandu, Nepal

E-mail: anita2grg@gmail.com to its minimal post-operative complications and preservation of vital structures unlike enucleation. ${ }^{1}$ This procedure involves removing the roof of the lesions that will release the intramural pressure and favor the formation of new bone. However, this approach results in a post-surgical defect in the form of an intra oral opening. This opening needs to be maintained, so that bone is deposited within the cyst cavity and the cyst will ultimately decrease significantly in size. Therefore, maintenance of the surgical opening before the bony infill occurs, is a critical determinant for the success of marsupialization. ${ }^{2-5}$

There are various approaches to maintaining the patency of the post-surgical defect, namely iodoform gauze packing, decompression stents and obturator prosthesis. ${ }^{3}$ Among these methods, obturators are more hygienic and also do not 
require frequent replacement unlike gauze packing. Depending on the phase of treatment, obturators used for post-surgical defect can be classified as: (i) Surgical obturator, (ii) Interim obturator and (iii) Definitive obturator. For the post-surgical defect following marsupialization, the interim obturator serves as a provisional prosthesis to further aid in the closure of the defect after initial healing. Taking into consideration the teeth missing, the anteroposterior position of the lesion, and the direction of marsupialization, there are three different obturator designs that have been introduced, namely: (i) Clasp type, consisting of a retainer and obturator; (ii) Denture type, consisting of a retainer, artificial tooth, denture base, and obturator; and (iii) Plug type, consisting only of an obturator. ${ }^{3}$

This article describes the fabrication of a clasp retained plug type interim obturator for the prosthetic management of a case with an acquired mandibular defect following marsupialization of an odontogenic tumor in the left posterior mandibular region.

\section{Case Report}

An 18-year old female patient reported to the Department of Prosthodontics and Maxillofacial Prosthetics, who was referred from the Department of Oral and Maxillofacial Surgery, People's Dental College and Hospital, for the fabrication of a mandibular obturator. History revealed that marsupialization was performed in the left mandibular ramus which had resulted in a defect extending in the buccal region of 36 and 37 . For the past two months, irrigation with normal saline and placement of betadine gauze pack was done daily, after which the patient presented to our department for the fabrication of obturator.
A thorough medical and dental history of the patient was recorded and an orthopantomograph (OPG) was taken, which revealed radiolucent lesion surrounding an impacted 38 (Fig 1). The extra oral examination revealed a large diffuse, non-tender swelling on the left cheek region (Fig 2). On the other hand, the intraoral examination revealed a post-surgical defect in the form of an intra-oral opening in the mandibular buccal vestibular region of 36 and 37 with the dimensions of $15 \mathrm{~mm}$ length, $6 \mathrm{~mm}$ breadth and $12 \mathrm{~mm}$ depth (Fig 3)

After removal of the betadine gauze pack and cleaning of the defect with betadine solution, the impression was made using irreversible hydrocolloid impression material in a stock tray (Fig 4) and a master cast was poured in Type III dental stone. Wax up was done, involving the lingual surface extending from 37 to 47 and also partially in the defect area. A clasp was fabricated and modified to adapt between 36 and 37 and also serve to connect the plug and the lingual plate (Fig 5). Processing was done to fabricate the obturator with heat cure acrylic resin. The next day, the completed obturator was tried in patient's mouth, and adjustments were made for proper extension and comfort of the patient and delivered to the patient (Fig 6) with instructions regarding its use and maintenance.

One week after the prosthesis delivery, the patient came for her first follow up. The patient was well adjusted to the prosthesis with no complains. No adjustments were required. The next follow up was done 1 month later (Fig 7). As the defect healed, the floor of the defect pushed the obturator outwards, hence the obturator was trimmed in this visit to allow further healing of the defect. The patient was then recalled every month to assess the healing. 


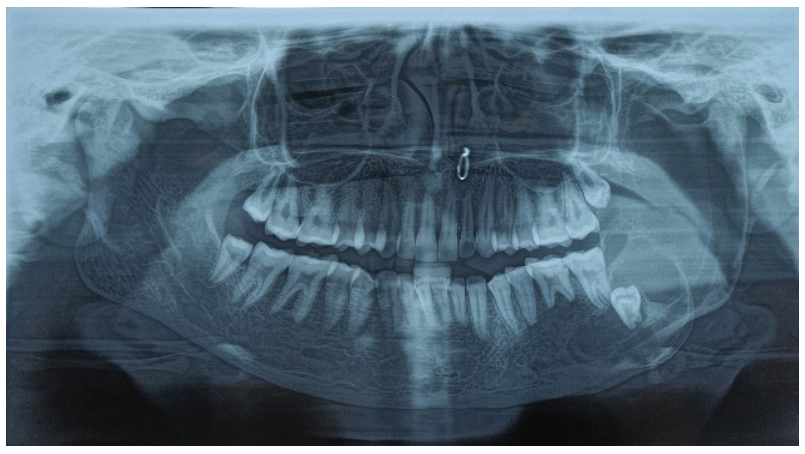

Figure 1: Orthopantomogram

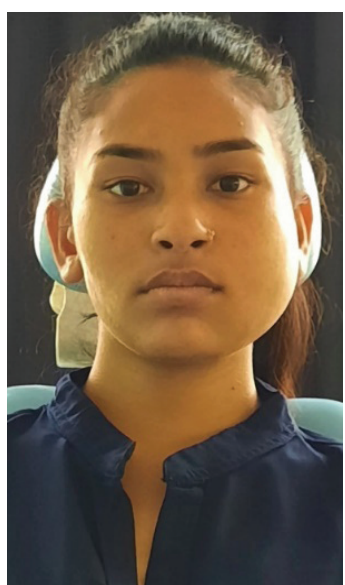

Figure 2: Clinical Presentation

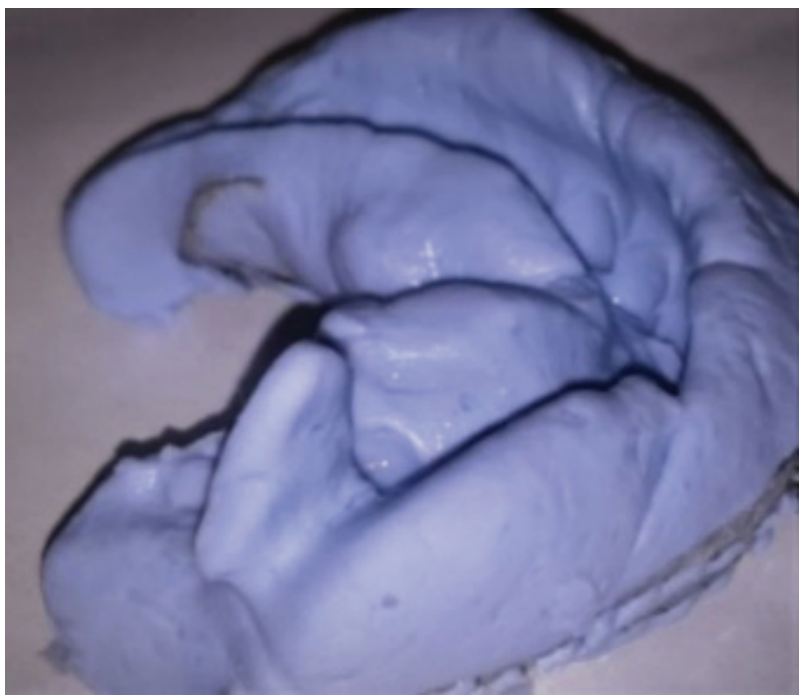

Figure 4: Irreversible hydrocolloid impression

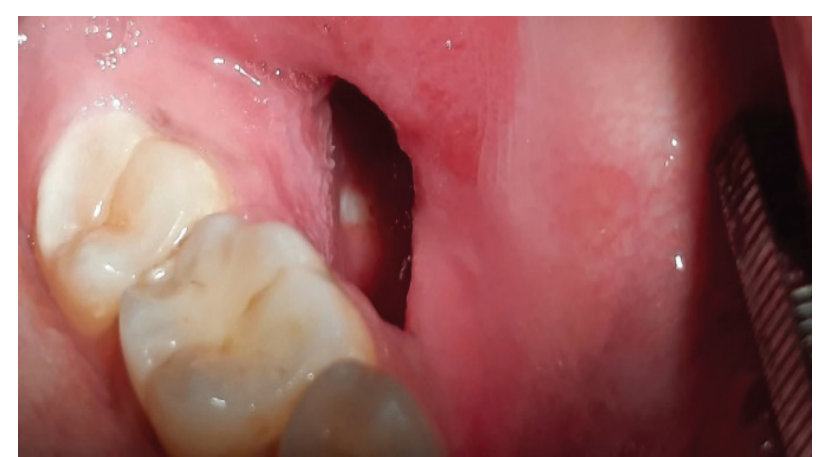

Figure 3:Post-surgical defect

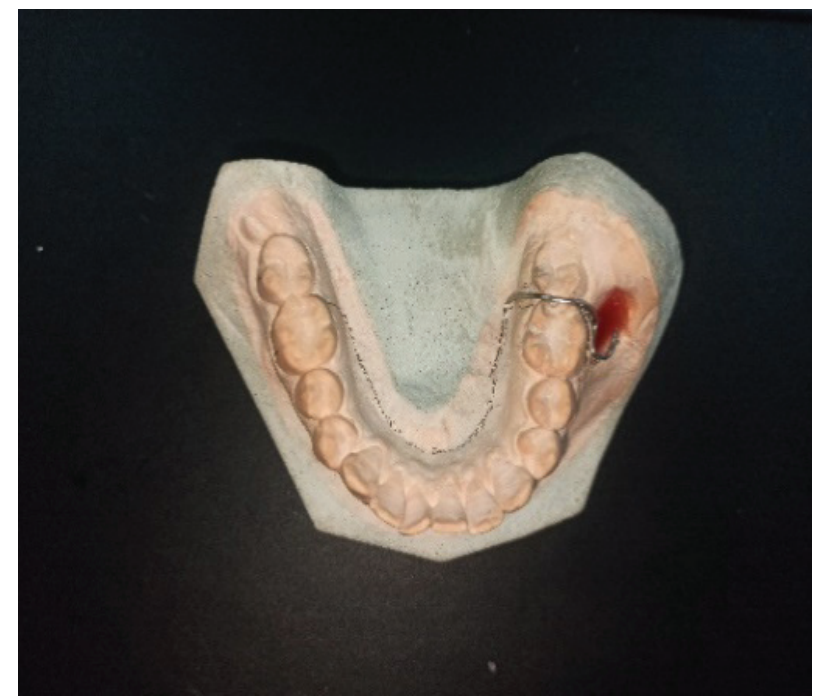

Figure 5:Clasp adapted between 36 and 37 


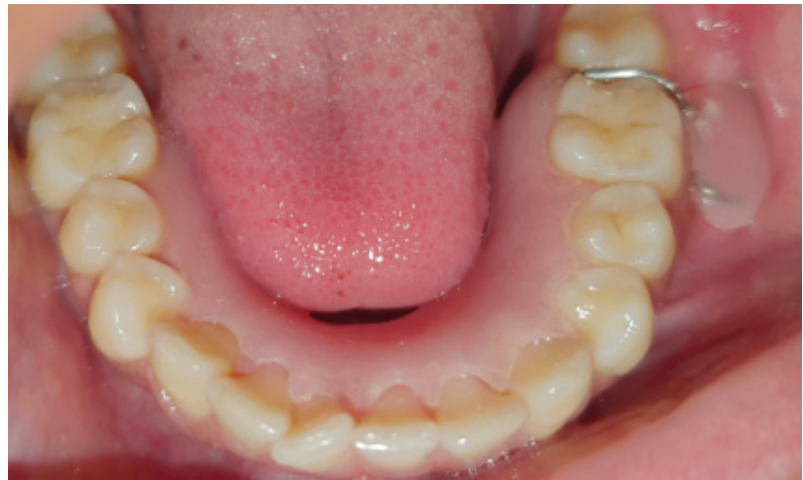

Figure 6: Insertion of the obturator

\section{Discussion}

After marsupialization of benign tumors and jawbone cysts, insertion of an obturator prosthesis is essential to maintain the surgical opening till complete closure of the defect. It has various clinical advantages that include fewer visits to the clinic to replace absorbent gauze, prevent accumulation of food into the lesion, and improved hygiene in the area. ${ }^{3}$

In the case described in this report, marsupialization was performed as a conservative surgical approach without the extraction of the impacted tooth due to various factors: (i) young age of the patient (ii) large size and extent of the lesion and (iii) the deep position of the impacted third molar which was approximating the inferior alveolar nerve canal. The resultant post-surgical defect was then protected with a plug type of obturator, retained with a clasp which serve to connect the defect on the buccal region and the lingual surface of the prosthesis, (Fig 6) and hence achieve better retention and stability of the prosthesis. ${ }^{6}$ The prosthesis was fabricated using heat-cured acrylic, hence eliminating the harmful effect of the residual monomer of the material.

Post-insertion instructions about the importance for the continuous wear of the obturator and a regular one-month follow up should be well explained to the patient. The follow-ups are important for radiological, surgical and

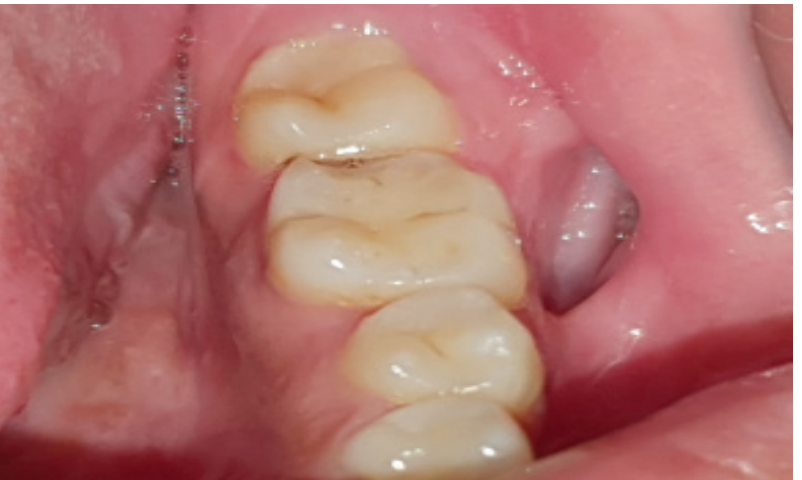

Figure 7: Examination during follow up

prosthetic review. From a prosthetic stand point, with the bone regeneration and a decrease in cavity size, the length of the obturator should be reduced while maintaining the diameter at the opening. The final decision to terminate the use of the obturator is done by the oral surgeon based on the radiological findings. ${ }^{3}$

However, the limitations in regard to the clasp and denture retained obturator was the need for adjustment in the interdental area between 36 and 37 for proper adaptation of clasp as well as to avoid impingement by the opposing tooth during occlusion, which indicated this type of obturator to be more invasive than the simple plug type obturator. ${ }^{3}$

\section{Conclusion}

The post-surgical defect, following marsupialization can be well maintained with the provision of an interim obturator prosthesis in the form of an acrylic plug. Addition of clasp can enhance the retention and stability of such prosthesis.

\section{References}

1. Martin Woods, Peter A. Reichart, 87 - Surgical Management of Nonmalignant Lesions of the Mouth, Editor(s): Peter A. Brennan, Henning Schliephake, G.E. Ghali, Luke Cascarini, Maxillofacial Surgery (Third Edition), Churchill Livingstone, 2017, Pages 1319-1334, ISBN 9780702060564. 
2. Taicher S, Steinberg H, Lewin-Epstein J, Sela M. Acrylic resin stents for marsupialization. J Prosthet Dent. 1985 Dec;54(6):818-9.

3. Murakami M, Nishi Y, Nishio M, Minemoto Y, Shimizu T, Nishimura M. A Retrospective Cohort Study of the Cumulative Survival Rate of Obturator Prostheses for Marsupialization. J Prosthodont. 2019 Feb;28(2):e811-e816.

4. Mubashir AS, Motwani BK, Sahu SK, Singh S, Dani A, Kulkarni S. Management of Post Surgical Defect in the Mandible with an Interim
Obturator: A Case Report. Int J Oral Health Med Res 2015;2(1):42-44.

5. Ghandour L, Bahmad HF, Bou-Assi S. Conservative Treatment of Dentigerous Cyst by Marsupialization in a Young Female Patient: A Case Report and Review of the Literature. Case Rep Dent. 2018 Jun 28;2018:7621363.

6. Hussain M, Hassan SI, Naqvi SK, Khan MY, Khan M, Tanveer W. Retention in mandibular obturators. Professional Med J 2014;21(4): 755759. 